\title{
Bridging Maladaptive Social Self-Beliefs and Social Anxiety:
}

\section{A Network Perspective}

\author{
Alexandre Heeren ${ }^{1,2}$, Emily E. Bernstein ${ }^{3}$, and Richard J. McNally ${ }^{3}$ \\ ${ }^{1}$ Psychological Sciences Research Institute, Université catholique de Louvain \\ ${ }^{2}$ Institute of Neuroscience, Université catholique de Louvain \\ ${ }^{3}$ Department of Psychology, Harvard University
}

\section{The final publication is available at https://doi.org/10.1016/i.janxdis.2020.102267}

Please cite as follows: Heeren, A., Bernstein, E.E., \& McNally, R.J. (2020). Bridging maladaptive social self-beliefs and social anxiety: A network perspective. Journal of Anxiety Disorders, 74: 102267. https://doi.org/10.1016/j.janxdis.2020.102267

\section{Author Note}

Alexandre Heeren iㅗ https://orcid.org/0000-0003-0553-6149

Emily E. Bernstein (i) https://orcid.org/0000-0001-8609-3153

Richard J. McNally ib https://orcid.org/0000-0002-5228-8777

The de-identified data and R code are publicly available via the Open Science Framework and can be accessed at https://osf.io/pn9ma/.

We have no known conflict of interest to disclose.

Correspondence concerning this article should be addressed to Alexandre Heeren, Psychological Sciences Research Institute, Université catholique de Louvain (Office E215), 10 Place du Cardinal Mercier, 1348 Louvain-la-Neuve, Belgium. Email: alexandre.heeren@uclouvain.be 


\begin{abstract}
The Clark and Wells (1995) model of social anxiety disorder postulates that three types of maladaptive social self-beliefs (high standard, conditional, and unconditional beliefs) play a crucial role in the development of fear and avoidance of social-evaluative situations-i.e., the hallmark symptoms of social anxiety disorder. In this project, we examined associations between the three types of maladaptive social self-beliefs and fear and avoidance of socialevaluative situations in a nonclinical community sample $(n=389)$. We used network analysis to estimate functional relations among aspects of maladaptive self-beliefs, fear, and avoidance and computed two different network models, a graphical Gaussian model (GGM) and a directed acyclic graph (DAG). Each model estimates edges and the importance of nodes in different ways. Both GGM and DAG pointed to fear and conditional beliefs as especially potent bridges between maladaptive social self-beliefs and social anxiety in our nonclinical sample. Altogether, these results offer data-driven heuristics in the field's larger, ongoing effort to illuminate pathways at play in the development of social anxiety. We situate this study within novel network approaches for developing theory-driven models and tests of the instigation and interactions of maladaptive social self-beliefs and social anxiety. However, because this is the first study to combine GGM and DAG in social anxiety research, we also discussed the caveats to this approach to help to usher the field forward.
\end{abstract}

Keywords: Fear, Avoidance, Social anxiety disorder, Maladaptive social self-beliefs, Probabilistic dependencies, Network analysis, Directed acyclic graph 


\section{Introduction}

Over the last 20 years, numerous cognitive theories have been proposed to account for the development and maintenance of fear and avoidance of social-evaluative situations - i.e., the hallmark symptoms of social anxiety disorder (SAD; e.g., Clark \& Wells, 1995; Heimberg et al., 2010; Rapee \& Heimberg, 1997; for a review, see Wong \& Rapee, 2016). A common feature of these theories is the emphasis on maladaptive self-beliefs (for reviews, see Gkika, et al., 2018;

Gregory et al., 2016; Wong \& Rapee, 2016). Drawing heavily on the influential Clark and Wells (1995) model of SAD, most of these models hold that individuals with social anxiety possess problematic beliefs about themselves and their social world (e.g., "I have to convey a favorable impression"; "People think I am inferior"; "If people know I am anxious, they will think I am weak"). And, from this perspective, these problematic beliefs are assumed to render these individuals likely to appraise social situations as more threatening than they actually are, thereby motivating fearful situational avoidance and potentially climaxing in full-blown SAD (e.g., Clark \& Wells, 1995; Gregory et al., 2016; Wong \& Rapee, 2016).

According to Clark and Wells (1995), three types of social self-beliefs may especially strengthen fear and avoidance: (a) high standards for social performance (e.g., "I must be able to convey a favorable impression to everyone"), (b) conditional beliefs regarding social evaluation (e.g., "If people see I'm anxious, they'll think that I'm weak"), and (c) unconditional beliefs about the self (e.g., "People think badly of me"). Exploratory and confirmatory factor analyses have identified such a three-factor solution as the best-fitting model of maladaptive social selfbeliefs in both clinical and nonclinical samples (e.g., Maeda et al., 2017; Wong \& Moulds, 2009; 2011a; Wong et al., 2014). 
However, uncertainty remains regarding how these three types of self-beliefs interact with fear and avoidance of social-evaluative situations. Despite studies reporting moderate-tolarge positive correlations between the distinct social self-beliefs and the severity of social anxiety in clinical and nonclinical samples (for a review, see Gkika et al., 2017), research focusing on their specific associations with fear and avoidance — and not merely on a sum score from a social anxiety questionnaire — is rare. The sole relevant study indicated that conditional self-beliefs predicted social avoidance in a nonclinical sample (Wong \& Moulds, 2011b). However, the authors did not consider the predictive relevance of avoidance for maladaptive self-beliefs. This is unfortunate as it is easy to envision how these maladaptive self-beliefs might interact with fear and avoidance ${ }^{1}$ (e.g., avoidance of social situation prevents disconfirmation of maladaptive social self-beliefs, which, in turn, fosters social fear). Likewise, uncertainty remains about the possible interactions between the distinct types of social self-beliefs (e.g., the higher one's standards and the stronger one's maladaptive unconditional beliefs). The complex, multifaceted interplay between maladaptive social self-beliefs and fear and avoidance of socialevaluative situations thus requires novel analytical approaches allowing one to map in a datadriven way the simultaneous connections among these variables. To do so, we estimated graphical network models to visualize and quantitatively consider the intricate relationships among maladaptive social self-beliefs and fear and avoidance of social-evaluative situations.

For decades, science has attempted to understand the world by boiling concepts down to their simplest components in a quest for reductionism, but there is now a move to map the complexities of the world via a network perspective (Barabási, 2012). Clinical psychology research has embraced this perspective as well, conceptualizing mental disorders as network systems wherein nodes denote symptoms and the edges the associations between them. In this 
way, instead of investigating a construct as unitary and formed from multiple variables, a network approach allows an investigation into the structure of, and associations between, the variables themselves. Though only recently pioneered by Borsboom and his colleagues (e.g., Borsboom et al., 2011, Borsboom \& Cramer, 2013), this approach has quickly become a hot topic in the contemporary clinical psychology. And, many studies have accordingly used this framework to investigate the interrelations between systems of symptoms and speculate as to the clinical implications (for systematic reviews, see Contreras et al., 2019; Robinaugh et al., 2019).

In clinical psychology, most network analyses have elucidated associations (“edges”) between psychiatric symptoms ("nodes"; for a review, see Contreras et al., 2019; Robinaugh et al., 2019); thereby enabling researchers to visualize disorders as complex network systems in a data-driven manner. With regard to social anxiety, three studies confirmed that SAD symptoms can indeed be viewed as a network system of interacting symptoms of fear and avoidance of social-evaluative situations (Heeren et al., 2018a; Heeren \& McNally, 2018a; Rodebaugh et al., 2018).

Network analysis, however, has not been confined to symptoms of mental disorders. Researchers have recently used network analysis to study cognitive and behavioral processes of mental disorders (e.g., Bernstein, et al., 2017, 2019; Heeren \& McNally, 2016; Hoorelbeke et al., 2016; Kraft et al., 2019; for a discussion, see Jones et al., 2017). In our prior research (Heeren \& McNally 2016), we have accordingly started to expand network approaches beyond SAD symptoms to include non-symptoms processes (i.e., laboratory measures of attentional bias for threat and attention control). In this way, we found that fear and avoidance of social situations remained the most influential variables in determining the entire network flow among attentional processes presumably involved in the maintenance of SAD in a sample of patients with SAD. 
Given how Clark and Wells (1995) postulated a highly influential role for the three types of maladaptive social self-beliefs in the development of social anxiety, clarifying the associations between these beliefs and fear and avoidance of social situations in a nonclinical sample is of critical interest as it may illuminate potential pathways to SAD. In this project, we therefore extended our previous line of research by examining in a nonclinical sample the associations among, on the one hand, fear and avoidance of social situations and, on the other hand, theorydriven core non-symptom maladaptive social self-beliefs, as emphasized by Clark and Wells (1995). By construing these beliefs as nodes, we aimed to visualize their model of social anxiety as a psychological network.

We had three primary goals. First, we endeavored to elucidate the relations between pairs of elements to disclose the pairwise connections among the types of maladaptive social selfbeliefs and fear and avoidance of social situations. Of critical interest was the computation of centrality metrics that disclose the most potentially influential nodes maintaining the network structure (Borgatti, 2005). Second, we tested whether these nodes cohere as a unitary network of interacting elements or constitute distinct communities (or subnetworks) of nodes serving different functions and, if so, whether certain nodes function as bridges, i.e., processes that connect or are shared by communities. These analyses may offer data-driven heuristics — to be followed up on and rigorously tested - in the search for especially potent nodes that may foster broader vulnerability in the instigation of social anxiety, or the mutually reinforcing interactions between maladaptive social self-beliefs, fear, and avoidance. Third, we used Bayesian methods to compute a directed acyclic graph (DAG) to estimate the probabilistic dependencies among the different nodes. A DAG is a directed network whereby each edge has an arrow tip on one end, signifying the direction of probabilistic dependence (e.g., McNally, 2016; Pearl et al., 2016; 
Scutari, 2010). To do so, DAGs encode the conditional independence relationships and characterize the joint probability distribution of the variables (Moffa et al., 2017; Pearl et al., 2016). DAGs can thus be decomposed as a product of the conditional distribution of each node given its parent nodes in the graph, thus rendering DAG analysis capable of indicating whether the presence of node A (“downstream”) probabilistically implies the presence of node B ("upstream") more than vice versa (for more details regarding the logic behind the use of DAGs, see Pearl, 2009) $)^{2}$. Here, we therefore computed DAGs to generate a computationally driven model of the probabilistic dependencies among the different nodes. Altogether, these complementary approaches - i.e., GGM and DAG - can provide a novel perspective and motivate more data- and theory-driven, focused experimental work.

\section{Method}

\subsection{Open Practices and Data Sharing}

De-identified data as well as R code are publicly available via the Open Science Framework and can be accessed at https://osf.io/pn9ma/.

\subsection{Participants}

We recruited 389 Belgian French-speaking volunteers (285 women, 73.3\%) from the general community via media and listserv advertisements inviting people to participate to an online survey. In line with previous research on the relations between maladaptive social selfbeliefs and social anxiety (e.g., Wong \& Moulds, 2009), we relied on an unselected community sample to avoid potential problems of range restriction and maximize generalizability. Participants were between the ages of 19 and $76(M=32.59, S D=12.29)$. Their years of education completed since primary school ranged from 6 to $17(M=16.18, S D=1.93)$. The study was approved by the Institutional Review Board at the first author's university and 
conducted according to the Declaration of Helsinki. Each participant provided informed consent before completing the survey.

\subsection{Materials}

\subsubsection{Liebowitz Social Anxiety Scale (LSAS; Liebowitz, 1987)}

The LSAS is a widely used, 24-item scale that measures fear and avoidance of social and performance situations (e.g., returning goods to a store, talking with people you do not know very well, drinking while being observed). Participants rate each of the 24 social situations on a 4-point Likert-type scale, once for the intensity of fear (0, None; 1, Mild; 2 Moderate; 3 Severe) and once for the frequency of avoidance of the situation (0, Never; 1, Occasionally; 2 Often; 3 Usually). We used the validated French self-report version of the scale (Heeren et al., 2012). The internal reliability of LSAS was high in the present sample, with a Cronbach's alpha of .96 for the global scale score (.92 for the fear scale score and .93 for the avoidance scale score). Because of our interest in distinguishing the respective influence of fear and avoidance symptoms, we computed separate scores for fear and avoidance ratings accordingly.

\subsubsection{Self-Beliefs related to Social Anxiety scale (SBSA; Wong \& Moulds, 2009).}

The SBSA is a 15-item self-report questionnaire designed to measure the strength of beliefs about the self in a social context. We relied on the SBSA because it is the most common instrument for measuring social self-beliefs. It includes three subscales that map directly onto the three types of social self-beliefs proposed by Clark and Wells (1995): (a) four items measure excessively high standard beliefs (e.g., "I must get everyone’s approval"; "I have to appear intelligent and witty"); (b) seven items measure conditional beliefs (e.g., "If I don’t say something interesting, people won't like me"; "If people know I'm anxious, they will think I'm 
weak"); and (c) four items measure unconditional beliefs (e.g., "People think I'm boring"; “People don't respect me").

For each item, participants rate their current strength of agreement with the belief on an 11-point Likert-type scale, from 1 (Do not agree at all) to 11 (Strongly agree), with higher scores indicating greater endorsement of the maladaptive self-belief. Scores on the SBSA have excellent internal consistencies and good validity in both clinical and nonclinical samples (Wong \& Moulds, 2009; Wong et al., 2014). We used the validated French self-report version of this scale (Heeren et al., 2014). In the present study, the internal reliability of SBSA was high, with a Cronbach's alpha of .91 for the global scale score (.76 for the high standard beliefs scale score, .89 for the conditional beliefs scale score, and .80 for the unconditional beliefs scale score). Accordingly, we computed separate scores for the three subscales.

\subsection{Network Analyses}

To best capture the relations among fear, avoidance, and the three distinct types of maladaptive social self-beliefs, we computed two different network models, each estimating edges and the importance of nodes in different ways.

\subsubsection{Data Preparation.}

Although none of the variables violated normality according to benchmarks of skewness $>2$ and/or kurtosis $>7$ (Curran et al., 1996), we followed guidelines in psychological network analyses (Epskamp \& Fried, 2018) and applied the nonparanormal transformation via the R package huge (Jiang et al., 2019).

Given the presence of variables with possible conceptual overlap (e.g., fear and avoidance scores of the LSAS; for a discussion, see Heeren \& McNally, 2018b), we used a datadriven method for identifying potentially redundant nodes among our five variables (i.e., fear, 
avoidance, and the three social self-beliefs) prior to computing the networks. First, we confirmed that our correlation matrix was positive definite, thereby confirming that nodes are not linear combinations of other nodes. Second, we searched for potential pairs of redundant nodes: ones that are highly intercorrelated $(r>0.50)$ and that exhibit highly similar patterns of correlations with the remaining nodes in the network (i.e., $>75 \%$ of correlations with other variables did not significantly differ for a given pair). To identify any such functionally indistinguishable pairs of nodes, we implemented the Hittner method for comparing dependent correlations (Hittner et al., 2003) via the goldbricker function of the R package networktools (Jones, 2018). There were no apparent redundant variables in the present dataset.

\subsubsection{Graphical LASSO Network.}

We used a graphical Gaussian model (GGM) to estimate the first network. In this network, edges signify conditional independence relationships between nodes, controlling for the effects of all other nodes (Epskamp \& Fried, 2018). We present GGMs that were regularized via the graphical LASSO (Least Absolute Shrinkage and Selection Operator) algorithm, which serves two primary functions (Friedman et al., 2011). First, it computes regularized partial correlations between pairs of nodes, thereby eliminating spurious associations (edges) attributable to the influence of other nodes in the network. Second, it shrinks trivially small associations to zero, thereby removing potentially "false positive" edges from the graph and producing a sparse graph comprising only the strongest edges. We used the R package qgraph (Epskamp et al., 2012) that automatically implements the graphical LASSO regularization in combination with Extended Bayesian Information Criterion (EBIC) model selection (Foygel \& Drton, 2011). In this procedure, 100 models with varying degrees of sparsity are estimated; a final model is selected according to the lowest EBIC value, given a specific hyperparameter 
gamma $(\gamma)$, which controls the trade-off between including false-positive edges and removing true edges. The hyperparameter $\gamma$ is usually set between zero and 0.5 (Epskamp et al., 2018). The closer one chooses a value of $\gamma$ near 0.5 , the more the EBIC will favor a simpler model containing fewer edges, whereas the closer one chooses a value of $\gamma$ near zero, the more the EBIC will favor a model with more edges. We opted to set $\gamma$ to 0.5 to be confident that our edges are genuine, assuming that the true model is, indeed, sparse (Epskamp \& Fried, 2018). To estimate the stability of edge weights, we bootstrapped the confidence regions of the edge weights via nonparametric bootstrapping with replacement (Epskamp et al., 2018). We also used a bootstrapped difference test to identify significant differences between edge weights (Epskamp et al., 2018).

To quantify the importance of each node in the resulting graphical LASSO network, we computed expected influence centrality (Robinaugh et al., 2016). The expected influence of a node is the sum of the edge weights incident on a given node, including positive and negative values. Higher expected influence values indicate greater centrality and thus greater importance in the network. The plot depicts the normalized (z-scored) expected influence centrality values for each node. To ensure the stability of this centrality index, we performed a person-dropping bootstrap procedure (Costenbader \& Valente, 2003). We also used a bootstrapped difference test to identify significant differences between nodes in terms of centrality (Epskamp et al., 2018).

Finally, we tested whether maladaptive social self-beliefs and symptoms of fear and avoidance cohere as one or multiple subnetworks (“communities"). Following prior network research (e.g., Bernstein et al., 2019; Heeren et al., 2018b; Robinaugh et al., 2014), we implemented the spin glass algorithm (Reichardt \& Bornholdt, 2006), a modularity-based community detection procedure suitable for uncovering the structure of relatively small networks 
with negative edge values (e.g., Traag \& Bruggeman, 2009). We used the spinglass.community function $(\gamma=1$, start temperature $=1$, stop temperature $=.01$, cooling factor $=.99$, spins $=5)$ of the R package igraph (Csardi \& Nepusz, 2006). Nodes within a community are more strongly interconnected than they are with nodes in another community. Following previous studies (e.g., Bernstein et al., 2019; Heeren et al., 2018a), we also identified important nodes that serve as bridges between communities by computing the bridge expected influence index via the bridge function of the R package networktools (Jones, 2018). Bridge expected influence is the sum of the edge weights connecting a given node to all nodes in the other community or communities (Jones et al., 2019). The plot depicts the normalized (z-scored) values for ease of comparison and interpretation. This index identifies nodes that, when activated, are most likely to activate nearby communities (subnetworks; Jones et al., 2019).

\subsubsection{Directed Acyclic Graph (DAG).}

We then computed a DAG to estimate a directed, potentially causal structure of the relations between nodes based on their probabilistic dependencies (Pearl et al., 2016). To do so, we used a Bayesian hill-climbing algorithm implemented via the R package bnlearn (Scutari, 2010). As implemented by bnlearn, the bootstrap function computes the structural aspects of the network model by adding edges, removing them, and reversing their direction to ultimately optimize the goodness-of-fit target score, i.e., the Bayesian Information Criterion (BIC). This involves an iterative process of randomly restarting this process with different possible edges linking different node pairs, perturbing the system, and using 50 different random restarts to avoid local maxima. Following previous studies (e.g., Bernstein et al., 2017, Blanchard et al., 2020; McNally et al., 2017), we performed 100 perturbations (i.e., attempts to insert, delete, or 
reverse an edge) for each restart. As this iterative procedure unfolds, the function returns the best fitting network based on this random restart/perturbation procedure.

To ensure the stability of the resultant DAG, we then bootstrapped 10,000 samples (with replacement), computed a network for each sample, and averaged across the resulting networks to produce a final network structure, involving a two-step procedure. First, we determined how frequently a given edge appeared in the 10,000 bootstrapped networks. We then used the optimal cut-point method of Scutari and Nagarajan (2013) for retaining edges, which yields networks having both high sensitivity and high specificity. Second, we ascertained the direction of each surviving edge in the 10,000 bootstrapped networks. If a given edge pointed from node $\mathrm{X}$ to node $\mathrm{Y}$ in at least $51 \%$ of the bootstrapped networks, then this direction was represented in the final network. In summary, we first determined the structure of the network (i.e., whether an edge is present or not), and then determined the direction of each surviving edge.

We then visualized the averaged network in two ways. In the first visualization, edge weights (i.e., thickness) represent relative BIC values. Higher values and therefore thicker edges indicate greater importance to the network structure. In other words, removing a thick edge from the network would be more damaging to the model fit than removing a thin edge (McNally et al., 2017). In the second visualization, edge weights represent directional probabilities. Higher values, and therefore thicker edges, indicate a greater likelihood that the edge points in the direction depicted. Thus, a thick edge pointing from node $\mathrm{X}$ to node $\mathrm{Y}$ appeared in a larger proportion of averaged 10,000 bootstrapped networks than a thin edge pointing from node $\mathrm{Y}$ to node Z.

\section{Results}


For each variable, mean, standard deviation, range, skewness, and kurtosis are available in the supplementary materials (see Table S1 in Supplementary Materials). An initial correlation matrix as well as a network visualization of the zero-order correlations are also available in the Supplementary Materials (see Figure S1 and Figure S2, respectively).

\subsection{Graphical LASSO Network}

Figure 1 depicts the resultant graphical LASSO network (i.e., regularized partial correlations). Node placement was determined by Fruchterman and Reingold's (1991) algorithm whereby nodes nearer to the center of the graph tend to have the strongest connections with other nodes. A thicker edge denotes a larger association. Green full edges represent positive regularized partial correlations, whereas red dashed ones represent regularized negative partial correlations. A few pairwise connections stand out: fear and avoidance; conditional beliefs and unconditional beliefs; high standards beliefs and unconditional beliefs; conditional beliefs and fear. The stability analyses of edge weights suggest that edges are fairly stable and that the strongest edges are significantly larger than most others (see Figure S3 \& Figure S4 in Supplementary Materials). Figure 2 includes estimates of the expected influence. Conditional self-beliefs yielded the greatest expected influence value. Among symptoms of social anxiety, fear was the most central one. The person-dropping bootstrap procedure indicated that expected influence estimates are highly stable (see Figure S5 in Supplementary Materials) ${ }^{3}$. Moreover, the bootstrapped difference test revealed that the most central nodes have significantly higher expected influence estimates than less central nodes (including conditional self-beliefs and fear as the significantly most central nodes; see Figure S6 in Supplementary Materials).

Finally, the spin glass algorithm detected two communities of nodes. One community comprised fear and avoidance-i.e., SAD symptoms. A second community comprised the three 
types of maladaptive social self-beliefs. Figure 3 depicts bridge centrality analysis. From the community of nodes denoting SAD symptoms, fear had the highest bridge expected influence. From the other community, conditional and unconditional self-beliefs had highest bridge expected influence values. The person-dropping bootstrap procedure revealed that bridge expected influence estimates were highly stable (see Figure S5 in Supplementary Materials) ${ }^{4}$. Moreover, the bootstrapped difference test confirmed the previous observations: the nodes denoting fear, conditional, and unconditional self-beliefs yielded significantly higher bridge expected influence than do other nodes (see Figure S7 in Supplementary Materials).

\subsection{DAG Network}

Figure 4A depicts the DAG arising from the averaging of the 10,000 bootstrapped networks whereby edge thickness signifies how important an edge is to the network structure; the thicker the edge, the more vital it is to model fit (McNally, 2016). Avoidance emerged at the top of the model (i.e., upstream node), and fear was probabilistically dependent on it - that is, people are more likely to experience social fears if they also avoid social situations than vice versa. In turn, conditional beliefs appear probabilistically dependent on fear, and high standards and unconditional beliefs were probabilistically dependent on conditional beliefs.

In Figure 4B, edge weights represent directional probabilities. Higher values indicate greater likelihood that the edge points in the direction depicted. The directional probabilities were modest. For instance, the value of the edge between avoidance and fear was .56 (i.e., the edge pointed from avoidance to fear in only $56 \%$ of the bootstrapped networks). The values of the connections between fear and conditional beliefs (.55), conditional and unconditional beliefs (.63), unconditional and high standard beliefs (.56), and conditional and high standard beliefs (.55) were likewise thin. 


\section{Discussion}

Maladaptive social self-beliefs presumptively play a crucial role in the onset and maintenance of fear and avoidance of social situations. In this cross-sectional study, we set out to clarify the functional relations among those processes in a nonclinical sample. To do so, we computed network analyses by using two distinct computational approaches to characterizing those relations: a graphical LASSO and a DAG.

Perhaps the most striking finding was the convergence across analyses in the pattern that conditional beliefs and fear were highly central in determining network topology and highly interconnected. First, conditional beliefs and fear emerged as the two most central nodes in the GGM. Second, community detection revealed that these highly central nodes appeared in two functionally distinct communities: one featuring the three types of maladaptive social self-beliefs and the other symptoms of fear and avoidance. And, from this latter community, fear exhibited the strongest bridge influence vis-à-vis the other community, especially via conditional beliefs. This pattern is thus suggestive of bridges whereby activation issuing from social anxiety may propagate to maladaptive social self-beliefs and vice versa. Note that this clustering into two functionally independent entities fully aligns with the idea that maladaptive social self-beliefs might not be exclusive to social anxiety (Maurage et al., 2013a; Renner et al., 2012). Finally, these observations were echoed in the DAG wherein the presence of conditional beliefs was probabilistically dependent on the presence of fear; therefore, bolstering our confidence that fear and conditional beliefs, and their interrelationship, are especially intriguing pathways for further study.

Although this pattern of results may appear at odds with the claim of Clark and Wells (1995) that maladaptive social self-beliefs drive fear and avoidance, they dovetail with later 
amendments of the original model (e.g., Clark, 2005; Hirsch \& Clark, 2004; Wong \& Rapee, 2016). For instance, the DAG suggests that people are unlikely to hold strong, conditional social self-beliefs unless they also experience social fears — a finding that aligns with Clark's (2005) amendment that the fear perceived during a social-evaluative situation may play a central role in the instigation of maladaptive social self-beliefs. On the other hand, as denoted by the modest directional probability value for the arrow pointing from fear to conditional beliefs (see Figure 4B), one cannot exclude that the direction of prediction between fear and conditional beliefs may go both ways (see our discussion below).

In the same vein, the central role of conditional beliefs is not surprising. It echoes research showing that conditional beliefs yield stronger associations with social anxiety than do high standards and unconditional beliefs (Wong \& Moulds, 2011a). As suggested by Wong and Moulds (2011a), the conditional beliefs (e.g., "If people see I am anxious, they will think that I am weak") inherently possess social elements and uncertainty (e.g., regardless of whether people actually detect one's anxiety) that may explain why these beliefs are more related to social anxiety than are the other two types.

Strikingly, the DAG suggests that people are unlikely to experience fear of social situations unless they also avoid them. Although our findings cannot directly speak to the maintenance of SAD per se given our nonclinical sample, this perspective dovetails with a longstanding behaviorist tradition considering avoidance as a core mechanism for maintaining fear in anxiety disorders (for reviews, see Hofmann \& Hay, 2018; Krypotos et al., 2015). For instance, drawing on the prominent Mowrer (1960)'s two-stage theory, several theorists of social anxiety emphasize that avoidance prevents recovery by thwarting opportunities for inhibitory learning by blocking access to information that is incompatible with threat-related beliefs (e.g., 
McManus et al., 2008). In this way, avoidance of social situations - and use of safety behaviors in social situations that the person cannot overtly avoid - may directly exacerbate fear of socialevaluative situations and, in turn, foster threat-related beliefs. Indeed, the structure of the DAG suggests that maladaptive social self-beliefs are less likely to manifest in the absence of related avoidant behavior. If this were confirmed in follow-up longitudinal and more experimental research, diminishing avoidance might thus deactivate other nodes, precipitating a downstream cascade of beneficial change in the maintenance of fear and, in turn, social self-beliefs.

The present study may yield other implications. One goal of clinical science is to identify prodromal signs of SAD instigation to aid prevention or early intervention. The network approach suggests that bridge nodes connecting processes of interest to clinical symptoms are worthy of more in-depth consideration (Jones et al., 2019). Because fear of social-evaluative situations and conditional beliefs are the two nodes exerting the strongest bridge influence, our findings invite the hypothesis that their initial co-occurrence may constitute such prodromal signals. On the other hand, the purpose of this study required the use of a group-level approach and may not generalize to network flow occurring within an individual requisite for clinical recommendations for a specific client (Fisher et al., 2018). Estimating idiographic network analyses from intensive time-series data of individual participant may provide a more finegrained approach to best identify targets for meaningful prevention and intervention (Fisher et al., 2017).

However, these promising results notwithstanding, our approach has limitations. First, our cross-sectional networks preclude causal inferences (Maurage et al., 2013b). Instead, our results can highlight viable hypotheses for focused study ${ }^{5}$ as the absence of an edge between two nodes does exclude a direct causal link between them (Moffa et al., 2017). Conversely, the 
presence of a partial correlation — as in the GGM — between nodes A and B would only be expected to be non-zero if A causes B, B causes A, there is a reciprocal relationship between A and $\mathrm{B}$, or both $\mathrm{A}$ and $\mathrm{B}$ cause a third variable in the network. Partial correlation networks are thus hypothesis-generating structures, indicative of potential causal effects (for a discussion, see Epskamp \& Fried, 2018; van Borkulo et al., 2016). And, DAGs are even more informative by virtue of being capable of estimating both the strength and direction of the probabilistic dependence between two variables, and the lack of an edge between two variables thus indicates improbable causal relationships (Moffa et al., 2017). Thus, this work helps to narrow down potential pathways of interest for guiding new work.

Second, when considering DAGs based on cross-sectional data, one must not confuse the direction of dependence with temporal antecedence (Bonchi et al., 2017; Pearl et al., 2016). DAGs encode conditional independence relationships and characterize the joint probability distribution of the variables (Moffa et al., 2017). DAGs can then be decomposed as a product of the conditional distribution of each node given its parent nodes in the graph, thus rendering DAGs capable of indicating whether the presence of node A probabilistically implies node B more than vice versa. However, it does not signify the temporal precedence of node B (Pearl, 2009). Hence, the DAG may provide clues about probabilistic dependence between the variables, but direction alone cannot be interpreted temporally. Of course, one may wonder about conditional probability's relevance if it cannot tell us something definitive about causation. Yet predictability is valuable even when causality remains elusive - for an in-depth discussion, see Yarkoni and Westfall's (2017) defense of atheoretical machine learning.

Third, DAGs, by definition, assume that relationships are directed and acyclic. However, one can easily gauge the extent of the directional flip-flopping in the DAG wherein 
the thickness of the arc signifies the probability that the edge points in the direction depicted but does not signify that the probability that the edge points in the other direction is zero (McNally et al., 2017). Here, most edges were thin, signifying frequent directional reversals, and possible cycles (see McNally et al., 2017). For instance, the edge pointed from avoidance to fear in only $56 \%$ of the bootstrapped networks, thus indicating that it pointed the other direction in $44 \%$ of the bootstrapped networks. The direction of prediction between fear and avoidance may thus go both ways. Graphical vector autoregressive modeling approaches on intensive time-series data may help at further unveiling bidirectional linear relationships (e.g., Aalbers et al., 2019). On the other hand, such a "linear" approach to network dynamics contrasts with complexity research involving non-linear dynamical system (Pierce \& Merletti, 2006). Recently developed computational tools capitalizing on differential equations for modeling mental disorders as nonlinear dynamical network systems may ultimately achieve this aim (Robinaugh et al., 2019). But, as García-Velázquez and her colleagues observed, "cyclic relations are inherently difficult to analyze, and thus it seems wise to start with robust findings on directional dependence and only then build toward more complex models" (García-Velázquez et al., 2020, p. 247).

Fourth, one cannot exclude the possibility that some of the non-zero edges in both GGM and DAG network models were artifacts due to conditioning on a collider variable (e.g., variables $\mathrm{B}$ in $\mathrm{A} \rightarrow \mathrm{B} \leftarrow \mathrm{C}$; Rohrer 2018). A collider is defined for a pair of variables as a third variable that is causally influenced by both variables (Pearl, 2009). The collider variable normally blocks the path, but when one statistically or through experimental design controls for it, a spurious association between A and C may arise (Pearl, 2009). This might reveal an (apparent) association between two variables, where none exists (for reviews, see Greenland, 2003; Munafò et al., 2018). Following prior research discussing the prevention of collider biases 
(e.g., Lee, 2012; Munafò et al., 2018), we checked that the associations between any pair of variables that remain in the GGM and DAG were already present as important edges without conditioning on them - that is, the zero-order correlations matrix. In line with the absence of a collider bias, each edge in the GGM and the DAG also appeared as a thick edge in the network depicting the zero-order correlations (see Figure S1 and Figure S2 in the Supplementary Materials).

Fifth, some commentators have questioned the suitability of centrality indices, partly because some have been unstable in cross-sectional and temporal networks (e.g., Bringmann et al., 2019). Notably, closeness and betweenness have been categorized as the problematic indices; expected influence is a more typically stable metric (e.g., Robinaugh et al., 2016). Moreover, both expected influence and bridge expected influence estimates were highly stable in the present study. Following Terluin et al. (2016), we also confirmed that differential node variance did not distort the values of the centrality metrics (see Supplementary materials).

Sixth, although some studies suggest that declines in the severity of nodes high on strength centrality or expected influence centrality are especially predictive of recovery from disorder (e.g., Boschloo et al., 2016; Papini et al., 2020), uncertainty remains regarding their value as therapeutic targets (e.g., Rodebaugh et al., 2018) especially because it may be challenging to deactivate central nodes without immediately affect other ones (e.g., Bernstein et al., 2019). Thus, it bears repeating that centrality estimates should not be overinterpreted and should be considered within the larger patterns of results.

Seventh, we computed separate scores for fear and avoidance LSAS-ratings because distinguishing fear from avoidance has significant conceptual and clinical relevance. However, the LSAS relied on 24 distinct social situations that are each assessed twice, once for the 
intensity of fear and once for the frequency of avoidance of the situation. And, as observed in previous research (i.e., Heeren \& McNally, 2018b; Rodebaugh et al., 2018), the generation of item-level network based on the LSAS can be problematic, because fear and avoidance itempairs of similar situations can correlate highly. To safeguard against this issue, we thus relied on sum-scores and not on single item scores of fear and avoidance item-pairs. However, a critical next step in future iterations would be to assess fear and avoidance via different measurement tools.

Finally, following previous research on social self-beliefs (e.g., Wong \& Moulds, 2009), our participants were from an unselected community sample. However, a central tenet of previous literature is that social self-beliefs figure prominently in the development of social anxiety, and are thus not confined to patients qualifying for a diagnosis of SAD. Moreover, our sample was heterogeneous, with $26.41 \%$ of participants reporting a total score above 56 at the LSAS (the cut-off score for SAD for the French version), enabling us to avoid potential problems of restricted range variability (see supplementary materials) that are common in clinical samples (e.g., Fried, 2016; Terluin et al., 2016). Nevertheless, future iterations would want to consider the network structure of maladaptive social self-beliefs, and SAD symptoms in people with SAD and at varying points in the development and course of illness.

In conclusion, the present study is not definitive, but it does highlight the utility of rethinking the core processes of the Clark and Wells (1995) model as nodes interacting within a network system. Like other network studies on cross-sectional data, this study fulfills a valuable niche, where preliminary data provide critical clues for larger, more definitive future efforts. 


\section{Acknowledgments}

Alexandre Heeren was supported by the FRS-FNRS Belgian Science Foundation (Grant

“1.C.059.18F") and the Helaers Foundation for Medical Research. These foundations did not exert any editorial influence over this article. 


\section{Footnotes}

${ }^{1}$ One may wonder about fear as distinct from avoidance - we are indebted to one of the reviewers of this paper for highlighting this issue. As Rachman (1978) observed in his book "Fear and Courage," discordance between fear and avoidance is not uncommon, especially in people in the middle range of fearfulness. Unlike fearless people, courageous ones experience fear but do not avoid. Discordance is especially dramatic in dangerous military situations, in novice skydivers, and novice speakers whose first public talks occur in graduate school.

${ }^{2}$ Pearl (2009) may appear obscure for readers who do not possess a strong background in probability theory. The popular science book of Pearl and Mackenzie (2018) provides simplified explanations and illustrations. Moreover, although DAGs have occasionally been used in clinical psychology (e.g., Bernstein et al., 2017; McNally et al., 2017), they have increasingly become popular in other fields, and especially in epidemiology and medicine where numerous illustrations are available (e.g., Fleischer \& Roux, 2008; Williams et al., 2018).

${ }^{3}$ We also calculated the centrality stability correlation coefficient (CS-coefficient) to quantify the effects of the person-dropping bootstrap procedure. The CS-coefficient represents the maximum proportion of participants that can be dropped while maintaining a $95 \%$ probability that the correlation between centrality metrics from the full data set and the subset data are at least .70. Based on a simulation study (Epskamp et al., 2018), a minimum CScoefficient of .25 (and preferably $\geq .50$ ) is recommended for interpreting centrality indices. In the present study, the CS-coefficients was .79 for the expected influence, suggesting that we can thus be confident in the stability of this metric in this study.

${ }^{4}$ The CS-coefficients was .59 for the bridge expected influence, suggesting that we can thus be confident in the stability of this metric in the present study.

${ }^{5}$ If cross-sectional research did not have the slightest bearing on causal relationships, then we would have to consign entirely one of Cronbach's (1957) two disciplines of psychology-i.e., experimental and correlationalto the ashcan of history, leaving us with only experimental psychology (for an in-depth discussion on the beneficial role of cross-sectional research in the advancement of psychology and the development of subfields like personality or developmental psychology, see Cronbach, 1957). 


\section{References}

Aalbers, G., McNally, R. J., Heeren, A., de Wit, S., \& Fried, E. I. (2019). Social media and depression symptoms: A network perspective. Journal of experimental psychology. General, 148(8), 1454-1462. https://doi.org/10.1037/xge0000528

Barabási, A.-L. (2012) The network takeover. Nature Physics, 8:14-16. https://doi.org/10.1038/nphys 2188

Bernstein, E. E., Heeren, A., \& McNally, R. J. (2017). Unpacking rumination and executive control: A network perspective. Clinical Psychological Science, 5, 816-826. https://doi.org/10.1177/2167702617702717

Bernstein, E.E., Heeren, A., \& McNally, R.J. (2019). Reexamining trait rumination as a system of repetitive negative thoughts: A network analysis. Journal of Behavior Therapy and Experimental Psychology, 63, 21-27. https://doi.org/10.1016/j.jbtep.2018.12.005

Blanchard, M. A., Roskam, I., Mikolajczak, M., \& Heeren, A. (2020). A Network Approach to Parental Burnout. Child Abuse and Neglect, In press.

Bonchi, F., Hajian, S., Mishra, B., \& Ramazzotti, D. (2017). Exposing the probabilistic causal structure of discrimination. International Journal of Data Science Analytics, 3, 1-21. https://doi.org/10.1007/s41060-016-0040-z

Borgatti, S.P. (2005). Centrality and network flow. Social Networks. 27, 55-71. https://doi.org/10.1016/j.socnet.2004.11.008

Borsboom, D., \& Cramer, O.J (2013) Network analysis: An integrative approach to the structure of psychopathology. Annual Review of Clinical Psychology, 9, 91-121. https://doi.org/10.1146/annurev-clinpsy-050212-185608 
Borsboom, D., Cramer, A. O. J., Schmittmann, V. D., Epskamp, S., \& Waldorp, L. J. (2011). The small world of psychopathology. PloS One, 6, e27407. https://doi.org/10.1371/journal.pone.0027407

Bringmann, L. F., Elmer, T., Epskamp, S., Krause, R. W., Schoch, D., Wichers, M., Wigman, J. T. W., \& Snippe, E. (2019). What do centrality measures measure in psychological networks? Journal of Abnormal Psychology, 128, 892-903. https://doi.org/10.1037/abn0000446

Clark, D. M. (2005). A cognitive perspective on social phobia. In W. R. Crozier \& L. E. Alden (Eds.), The essential handbook of social anxiety for clinicians (pp. 193-218). New York, NY, US: John Wiley \& Sons Ltd.

Clark, D. M., \& Wells, A. (1995). A cognitive model of social phobia. In R. Heimberg, M. Liebowitz, D. A. Hope, \& F. R. Schneier (Eds.), Social phobia: Diagnosis, assessment, and treatment (pp. 69-93). New York: Guilford Press.

Contreras, A., Nieto, I., Valiente, C., Espinosa, R., \& Vazquez, C. (2019). The study of psychopathology from the network analysis perspective: A systematic review. Psychotherapy \& Psychosomatics, 88, 71-83. https://doi.org/10.1159/000497425

Costenbader, E., \& Valente, T. W. (2003). The stability of centrality measures when networks are sampled. Social Networks, 25, 283-307. https://doi.org/10.1016/S0378$\underline{8733(03) 00012-1}$

Cronbach, L. J. (1957). The two disciplines of scientific psychology. American Psychologist, 12, 671-684.

Csardi, G., \& Nepusz, T. (2006). The igraph software package for complex network research. InterJournal Complex Systems, 1695. Retrieved from http://igraph.sf.net/ 
Curran, P. J., West, S. G., \& Finch, J. F. (1996). The robustness of test statistics to nonnormality and specification error in confirmatory factor analysis. Psychological Methods, 1, 1629. https://doi.org/10.1037/1082-989X.1.1.16

Elliott, H., Jones, P.J., Schmidt, U. (2020). Central symptoms predict post-treatment outcomes and clinical impairment in anorexia nervosa: A network analysis. Clinical Psychological Science, 8, 139-154. https://doi.org/10.1177/2167702619865958

Epskamp, S., Borsboom, D., \& Fried, E. I. (2018). Estimating psychological networks and their accuracy: A tutorial paper. Behavior Research Methods, 50, 195-212. https://doi.org/10.3758/s13428-017-0862-1

Epskamp, S., Cramer, A. O. J., Waldorp, L. J., Schmittmann, V. D., \& Borsboom, D. (2012). qgraph: Network visualizations of relationships in psychometric data. Journal of Statistical Software, 48, 1-18. https://doi.org/10.18637/jss.v048.i04

Epskamp, S., \& Fried, E. I. (2018). A tutorial on regularized partial correlation networks. Psychological Methods, 23, 617-634. https://doi.org/10.1037/met000016

Fisher, A. J., Medaglia, J. D., \& Jeronimus, B. F. (2018). Lack of group-to-individual generalizability is a threat to human subjects research. Proceedings of the National Academy of Sciences, 115, E6106-E6115. https://doi.org/10.1073/pnas.1711978115

Fisher, A. J., Reeves, J. W., Lawyer, G., Medaglia, J. D., \& Rubel, J. A. (2017). Exploring the idiographic dynamics of mood and anxiety via network analysis. Journal of Abnormal Psychology, 126, 1044. https://doi.org/10.1037/abn0000311

Fleischer, N. L., \& Roux, A. V. D. (2008). Using directed acyclic graphs to guide analyses of neighbourhood health effects: An introduction. Journal of Epidemiology \& Community Health, 62, 842-846. https://doi.org/10.1136/jech.2007.067371 
Foygel, R., \& Drton, M. (2011). Bayesian model choice and information criteria in sparse generalized linear models. arXiv:1112.5635, 1-37.

Fried, E. I. (2016, November 26). New paper: differential variability of variables drives network structure [Web log post]. Retrieved from https://psych-networks.com/new-paperdifferential-variability-items-drives-network-structure/

Friedman, J., Hastie, T., \& Tibshirani, R. (2011). glasso: Graphical lasso-estimation of Gaussian graphical models. R package version 1.8.

Fruchterman, T., \& Reingold, E. (1991). Graph drawing by force directed placement. Software: Practice and Experience, 21, 1129-1164.

García-Velázquez, R., Jokela, M., \& Rosenström, T. M. (2020). Direction of dependence between specific symptoms of depression: A non-Gaussian approach. Clinical Psychological Science, 8, 240-251. https://doi.org/10.1177/2167702619875410

Gkika, S., Wittkowski, A, \& Wells, A. (2018). Social cognition and metacognition in social anxiety: A systematic review. Clinical Psychology and Psychotherapy, 25:10-30. https://doi.org/10.1002/cpp.2127

Greenland, S. (2003). Quantifying biases in causal models: Classical confounding vs. colliderstratification bias. Epidemiology, 14, 300-306.

Gregory, B., Peters, L., Rapee, R.M., 2016. The self in social anxiety. In: Kyrios, M., Moulding, R., Nedeljkovic, M., Bhar, S.S., Doron, G., Mikulincer, M. (Eds.), The self in psychological disorders (pp. 91-101) Cambridge, UK: Cambridge University Press. 
Heeren, A., Bernstein, E.E., \& \& McNally, R.J. (2018a). Deconstructing trait anxiety: A network perspective. Anxiety, Stress, \& Coping, 31, 262-276. https://doi.org/10.1080/10615806.2018.1439263

Heeren, A., Jones, P.J., \& McNally, R.J. (2018b). Mapping network connectivity among symptoms of social anxiety and comorbid depression in people with social anxiety disorder. Journal of Affective Disorders, 228, 75-82. https://doi.org/10.1016/j.jad.2017.12.003

Heeren, A., Maurage, P., Rossignol, M., Vanhaelen, M., Peschard, V., Eeckhout, C., \& Philippot, P. (2012). The self-report version of the Liebowitz Social Anxiety Scale: Psychometric properties of the French version. Canadian Journal of Behavioural Science, 44, 99-107. https://doi.org/10.1037/a0026249

Heeren, A. \& McNally, R.J. (2016). An integrative network approach to social anxiety disorder: The complex dynamic interplay among attentional bias for threat, attentional control, and symptoms. Journal of Anxiety Disorders, 42, 95-104. https://doi.org/10.1016/j.janxdis.2016.06.009

Heeren, A. \& McNally, R.J. (2018a). Social anxiety disorder as a densely interconnected network of fear and avoidance for social situations. Cognitive Therapy and Research, 42, 103-113. https://doi.org/10.1007/s10608-017-9876-3

Heeren, A., McNally, R.J. (2018b). Correction to: Social Anxiety Disorder as a Densely Interconnected Network of Fear and Avoidance for Social Situations. Cognitive Therapy and Research, 42, 878-879. https://doi.org/10.1007/s10608-018-9952-3

Heeren, A., Wong, Q.J.J., Ceschi, G., Moulds, M. L., \& Philippot, P. (2014). Probing the structural validity of the Self-Beliefs in Social Anxiety Scale (SBSA): Adaptation and 
validation in a French-speaking community sample. Canadian Journal of Behavioural Science, 46, 506-513. https://doi.org/10.1037/a0035295

Heimberg, R. G., Brozovich, F. A., \& Rapee, R. M. (2010). A cognitive model of social anxiety disorder: Update and extension. In S. G. Hofmann \& P. M. DiBartolo (Eds.), Social anxiety: Clinical, developmental, and social perspectives $\left(2^{n d}\right.$ Ed., pp. 395-422). New York, NY: Academic Press.

Hirsch, C. R., \& Clark, D. M. (2004). Information-processing bias in social phobia. Clinical Psychology Review, 24, 799-825. https://doi.org/10.1016/j.cpr.2004.07.005

Hittner, J. B., May, K., \& Silver, N. C. (2003). A Monte Carlo evaluation of tests for comparing dependent correlations. Journal of General Psychology, 130, 149-68. https://doi.org/10.1080/00221300309601282

Hofmann, S. G., \& Hay, A. C. (2018). Rethinking avoidance: Toward a balanced approach to avoidance in treating anxiety disorders. Journal of anxiety disorders, 55, 14-21. https://doi.org/10.1016/j.janxdis.2018.03.004

Hoorelbeke, K., Marchetti, I., De Schryver, M., \& Koster, E. H. W. (2016). The interplay between cognitive risk and resilience factors in remitted depression: A network analysis. Journal of Affective Disorders, 195, 96-104. https://doi.org/10.1016/j.jad.2016.02.001

Jiang, H., Fei, X., Liu, H., Roeder, K., Lafferty, J., Wasserman, L., Li, X., \& Zhao, T. (2019). huge: High-Dimensional Undirected Graph Estimation. R package version 1.3.2. https://CRAN.R-project.org/package=huge

Jones, P. J. (2018). Networktools: Tools for identifying important nodes in networks. R Package Version 1.2.2.

Jones, P.J., Heeren, A., \& McNally, R. J. (2017). Commentary: A network theory of mental 
disorders. Frontiers in Psychology, 8:1305. https://doi.org/10.3389/fpsyg.2017.01305

Jones, P. J., Ma, R., \& McNally, R. J. (2019). Bridge centrality: A network approach to understanding comorbidity. Multivariate Behavior Research, In Press. https://doi.org/10.1080/00273171.2019.1614898

Kraft, B., Jonassen, R., Heeren, A., Harmer, C., Stiles, T., \& Landrø, N.I. (2019). Attention bias modification in remitted depression is associated with increased interest and leads to reduced adverse impact of anxiety symptoms and negative cognition. Clinical Psychological Science, 7, 530-544. https://doi.org/10.1177/2167702618822480

Krypotos, A. M., Effting, M., Kindt, M., \& Beckers, T. (2015). Avoidance learning: a review of theoretical models and recent developments. Frontiers in Behavioral Neuroscience, 9, 189. https://doi.org/10.3389/fnbeh.2015.00189

Lee, J. J. (2012). Correlation and causation in the study of personality. European Journal of Personality, 26, 372-390. https://doi.org/10.1002/per.1863

Liebowitz, M. R. (1987). Social phobia. Modern Problems of Pharmacopsychiatry, 22, 141-173.

Maeda, S., Shimada, H., Sato, T., Tashiro, K., \& Tanaka, Y. (2017). Translation and initial validation of the Japanese version of the Self-Beliefs Related to Social Anxiety Scale. Psychological Reports, 120, 305-318. https://doi.org/10.1177/0033294116686037

Maurage, P., de Timary, P., Moulds, M. L., Wong, Q. J. J., Collignon, M., Philippot, P., \& Heeren, A. (2013a). Maladaptive social self-beliefs in alcohol-dependence: A specific bias towards excessive high standards. PLoS ONE, 8, (3), e58928. https://doi.org/10.1371/journal.pone.0058928 
Maurage, P., Heeren, A., \& Pesenti, M. (2013b). Does chocolate consumption really boost Nobel award chances? The peril of over-interpreting correlations in nutrition and health studies. Journal of Nutrition, 143, 931-933. https://doi.org/10.3945/jn.113.174813

McNally, R.J. (2016). Can network analysis transform psychopathology? Behaviour Research and Therapy, 86, 95-104. https://doi.org/10.1016/j.brat.2016.06.006

McNally, R.J., Heeren, A., \& Robinaugh, D.J. (2017). A Bayesian network analysis of posttraumatic stress disorder symptoms in adults reporting childhood sexual abuse. European journal of psychotraumatology, 8(sup3), 1341276. https://doi.org/10.1080/20008198.2017.1341276

McManus, F., Sacadura, C., \& Clark, D. M. (2008). Why social anxiety persists: an experimental investigation of the role of safety behaviours as a maintaining factor. Journal of Behavior Therapy and Experimental Psychiatry, 39, 147-161. https://doi.org/10.1016/j.jbtep.2006.12.002

Moffa, G., Catone, G., Kuipers, J., Kuipers, E., Freeman, D., Marwaha, S., . . Bebbington, P. (2017). Using directed acyclic graphs in epidemiological research in psychosis: An analysis of the role of bullying in psychosis. Schizophrenia Bulletin, 43, 1273-1279. https://doi.org/10.1093/schbul/sbx013

Morgan, H., \& Raffle, C. (1999). Does reducing safety behaviors improve treatment response in patients with social phobia? Australian and New Zealand Journal of Psychiatry, 33, 503510. https://doi.org/10.1080/j.1440-1614.1999.00599.x

Mowrer, O. H. (1960). Learning theory and behavior. New York, NY: Willey. 
Munafò, M. R., Tilling, K., Taylor, A. E., Evans, D. M., Smith, G. D. (2018). Collider scope: When selection bias can substantially influence observed associations. International Journal of Epidemiology, 47, 226-235. https://doi.org/10.1093/ije/dyx206

Papini, S., Rubin, M., Telch, M. J., Smits, J., \& Hien, D. A. (2020). Pretreatment posttraumatic stress disorder symptom network metrics predict the strength of the association between node change and network change during treatment. Journal of Traumatic Stress, 33, 6471. https://doi.org/10.1002/jts.22379

Pearce, N., \& Merletti, F. (2006). Complexity, simplicity, and epidemiology. International Journal of Epidemiology, 35, 515-519. https://doi.org/10.1093/ije/dyi322

Pearl, J. (2009). Causality: Models, reasoning and inference. Cambridge University Press: Cambridge, MA, USA.

Pearl, J., Glymour, M., Jewell, N. P. (2016). Causal Inference in Statistics: A Primer. Wiley.

Pearl, J., Mackenzie, D. (2018). The book of why: The new science of cause and effect. New York: Basic Books.

Rachman, S. J. (1978). Fear and courage._San Francisco, CA: Freeman.

Rapee, R. M., \& Heimberg, R. G. (1997). A cognitive-behavioral model of anxiety in social phobia. Behaviour Research and Therapy, 35, 741-756. https://doi.org/10.1016/s0005-7967(97)00022-3

Reichardt, J., \& Bornholdt, S. (2006). Statistical mechanics of community detection. Physical Review E, 74, 016110-016110-14. https://doi.org/10.1103/PhysRevE.74.016110

Renner F., Lobbestael J., Peeters F., Arntz A., Huibers M. (2012). Early maladaptive schemas in depressed patients: Stability and relation with depressive symptoms over the course of 
treatment. Journal of Affective Disorders, 136, 581-590.

https://doi.org/10.1016/j.jad.2011.10.027

Robinaugh, D., Haslbeck, J. M. B., Waldorp, L., Kossakowski, J. J., Fried, E. I., Millner, A., ... Borsboom, D. (2019). Advancing the network theory of mental disorders: A computational model of panic disorder. PsyArXiV. https://doi.org/10.31234/osf.io/km37w

Robinaugh, D. J., LeBlanc, N. J., Vuletich, H. A., \& McNally, R. J. (2014). Network analysis of Persistent Complex Bereavement Disorder in conjugally bereaved adults. Journal of Abnormal Psychology, 123, 510-522. https://doi.org/10.1037/abn0000002

Robinaugh, D. J., Millner, A. J., \& McNally, R. J. (2016). Identifying highly influential nodes in the complicated grief network. Journal of Abnormal Psychology, 125, 747-757. https://doi.org/10.1037/abn0000181

Rodebaugh, T. L., Tonge, N. A., Piccirillo, M. L., Fried, E., Horenstein, A., Morrison, A. S., .. . Heimberg, R. G. (2018). Does centrality in a cross-sectional network suggest intervention targets for social anxiety disorder? Journal of Consulting and Clinical Psychology, 86, 831-844. https://doi.org/10.1037/ccp0000336

Rohrer, J. M. (2018). Thinking clearly about correlations and causation: Graphical causal models for observational data. Advances in Methods and Practices in Psychological Science, 1, 27-42. https://doi.org/10.1177/2515245917745629

Scutari, M. (2010). Learning Bayesian networks with the BNLearn package. Journal of Statistical Software, 35, 1-22. https://doi.org/10.18637/jss.v035.i03 
Scutari, M., \& Nagarajan, R. (2013). Identifying significant edges in graphical models of molecular networks. Artificial Intelligence in Medicine, 57, 207-217. https://doi.org/10.1016/j.artmed.2012.12.006

Terluin, B., de Boer, M. R., \& de Vet, H. C. W. (2016). Differences in connection strength between mental symptoms might be explained by differences in variance: Reanalysis of network data did not confirm staging. PloS ONE 11(11): e0155205 https://doi.org/10.1371/journal.pone.0155205

Traag, V., \& Bruggeman, J. (2009). Community detection in networks with positive and negative links. Physical Review E, 80, 036115. https://doi.org/10.1103/PhysRevE.80.036115

van Borkulo, C. D., Borsboom, D, \& Schoevers, R.A. (2016). Group-level symptom networks in depression — Reply. JAMA Psychiatry, 73, 411-412. https://doi.org/10.1001/jamapsychiatry.2015.3157

Williams, T. C., Bach, C. C., Matthiesen, N. B., Henriksen, T. B., \& Gagliardi, L. (2018). Directed acyclic graphs: a tool for causal studies in paediatrics. Pediatric research, 84 , 487-493. https://doi.org/10.1038/s41390-018-0071-3

Wong, Q. J. J., \& Moulds, M. L. (2009). Impact of rumination versus distraction on anxiety and maladaptive self-beliefs in socially anxious individuals. Behaviour Research and Therapy, 47, 861-867. https://doi.org/10.1016/j.brat.2009.06.014

Wong, Q. J. J., \& Moulds, M. L. (2011a). A new measure of the maladaptive self-beliefs in social anxiety: Psychometric properties in a non-clinical sample. Journal of Psychopathology and Behavioral Assessment, 33, 285-297. https://doi.org/10.1007/s10862-010-9208-3 
Wong, Q. J. J., \& Moulds, M. L. (2011b). The relationship between maladaptive self-beliefs characteristic and social anxiety and avoidance. Journal of Behavior Therapy and Experimental Psychiatry, 42, 171-178. https://doi.org/10.1016/j.jbtep.2010.11.004

Wong, Q. J., Moulds, M. L., \& Rapee, R. M. (2014). Validation of the self-beliefs related to social anxiety scale: a replication and extension. Assessment, 21, 300-311. https://doi.org/10.1177/1073191113485120

Wong, Q.J.J., \& Rapee, R.M. (2016). The etiology and maintenance of social anxiety disorder: A synthesis of complimentary theoretical models and formulation of a new integrated model. Journal of Affective Disorders, 203, 84-100.

https://doi.org/10.1016/j.jad.2016.05.069

Yarkoni, T., \& Westfall, J. (2017). Choosing prediction over explanation in psychology: Lessons from machine learning. Perspectives on Psychological Science, 12, 1100-1122. https://doi.org/10.1177/1745691617693393 


\section{FIGURE CAPTIONS}

\section{Figure 1. Network constructed via the graphical LASSO.}

Note. The thickness of an edge reflects the magnitude of the association (the thickest edge representing a value of .52). Green full lines represent positive regularized partial correlations, whereas red dashed lines represent negative regularized partial correlations. Avoid = Avoidance of social situation; Fear = Fear of social situations; HighSt $=$ High standard beliefs; Cond= conditional beliefs; Uncond= unconditional beliefs.

\section{Figure 2. Expected influence estimates of the graphical LASSO}

Note . Avoid = Avoidance of social situation; Fear $=$ Fear of social situations; HighSt $=$ High standard beliefs; Cond= conditional beliefs; Uncond= unconditional beliefs .

\section{Figure 3. Bridge expected influence estimates of the graphical LASSO}

Note . Avoid = Avoidance of social situation; Fear $=$ Fear of social situations; HighSt $=$ High standard beliefs; Cond $=$ conditional beliefs; Uncond $=$ unconditional beliefs .

\section{Figure 4. Directed acyclic graphs (DAGs).}

Note. (A) Connection strength indicates in what percentage of the bootstrapped networks that connection appeared. Arrow thickness is drawn proportionately such that the thicker arrows indicate higher connection strength. (B) Direction probability indicates in what percentage of the bootstrapped networks the connection went in that direction. Arrow thickness is drawn proportionately such that the thicker arrows indicate higher direction probability. Avoid $=$ Avoidance of social situation; Fear $=$ Fear of social situations; HighSt $=$ High standard beliefs; Cond $=$ conditional beliefs; Uncond= unconditional beliefs. 


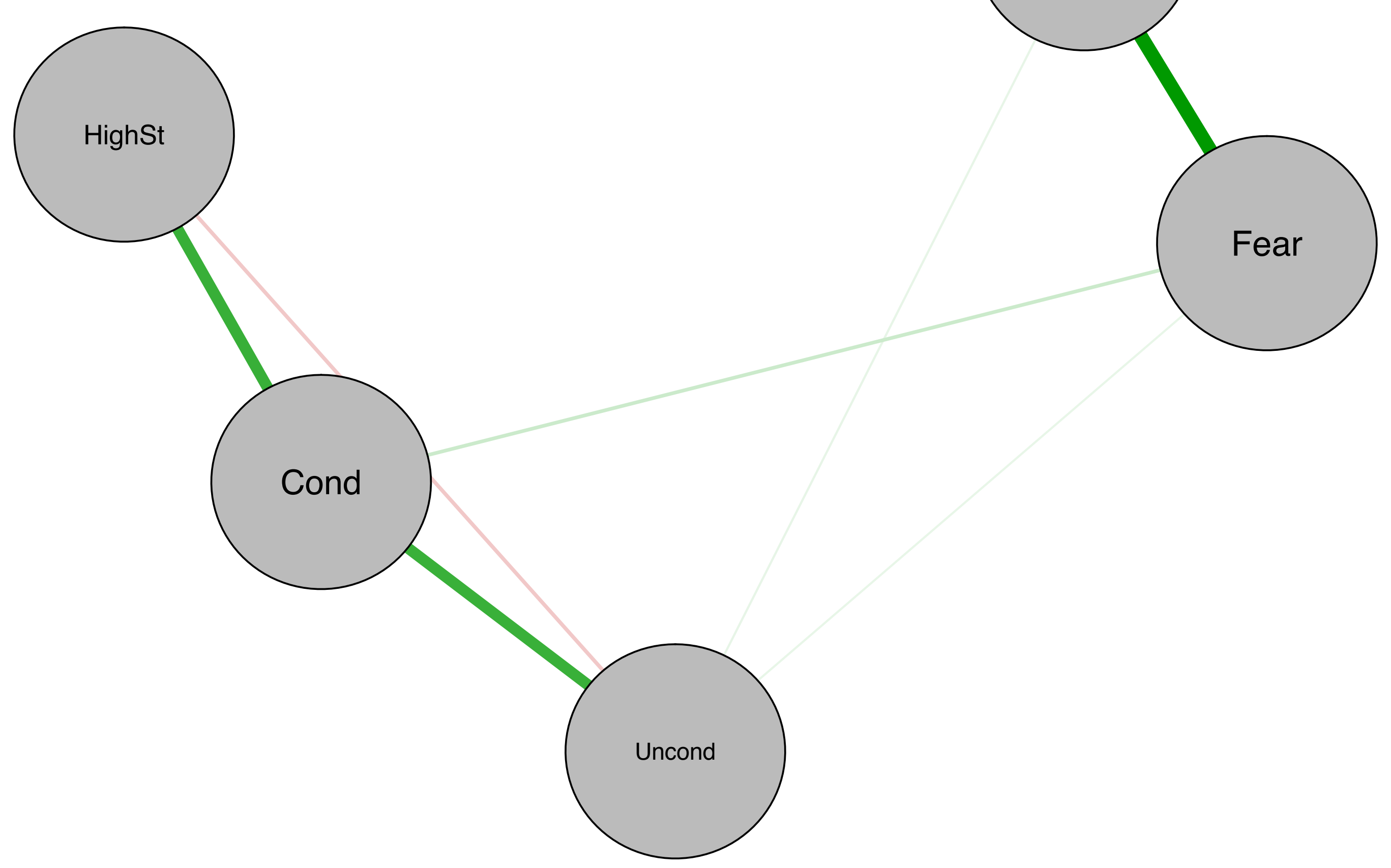




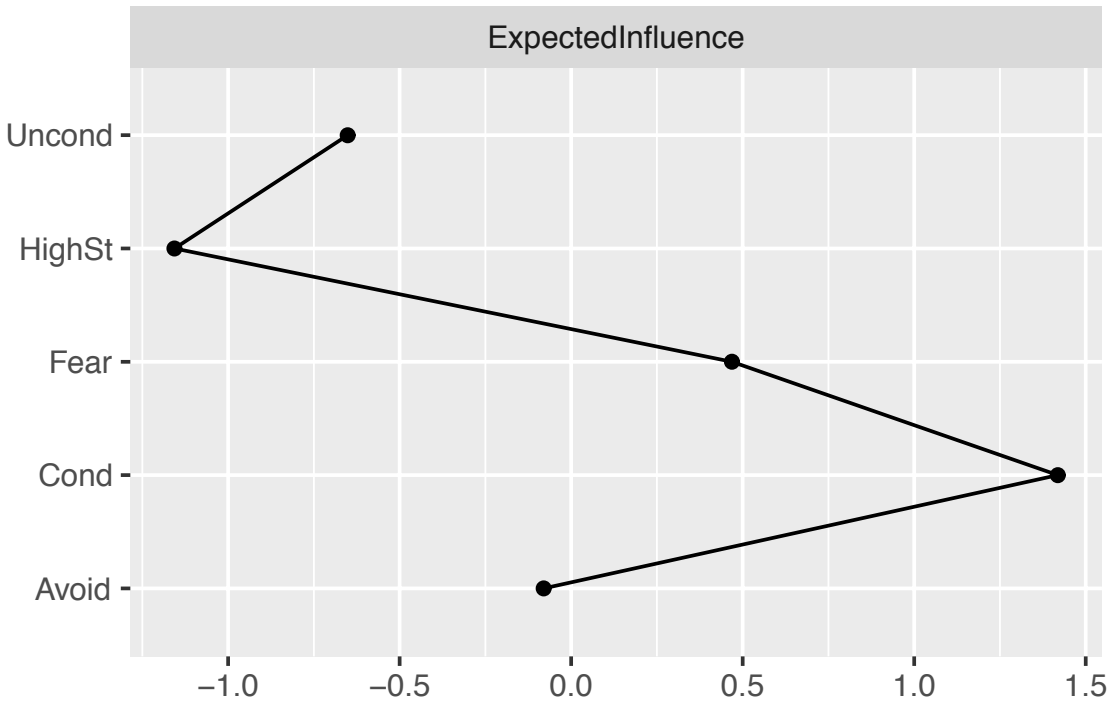




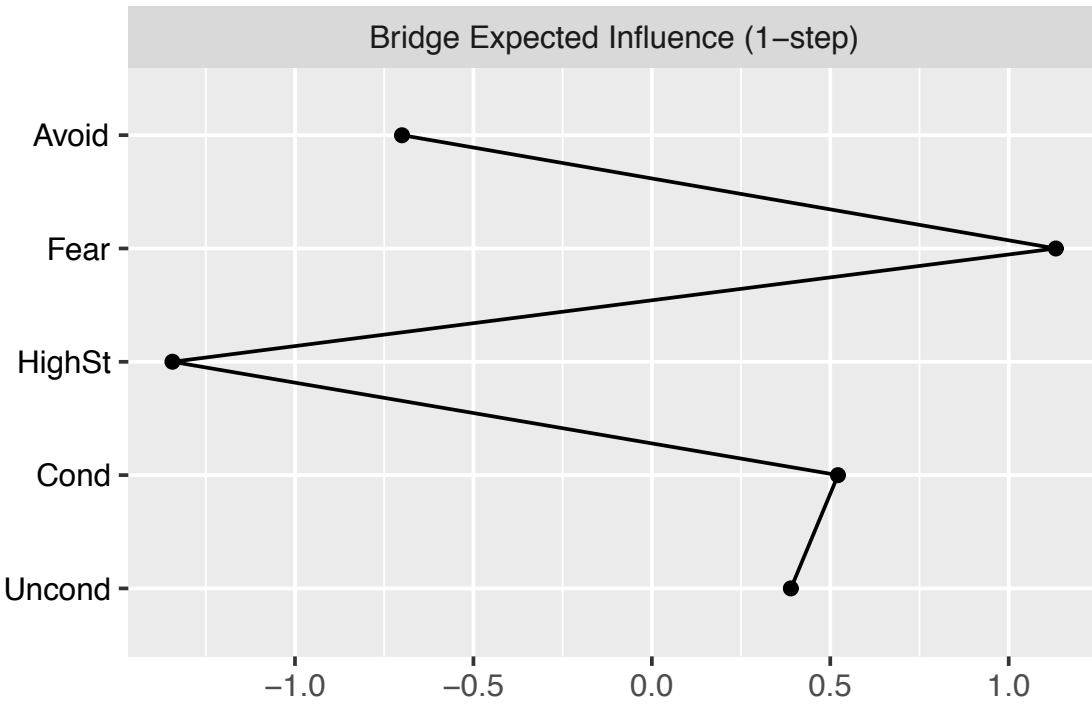



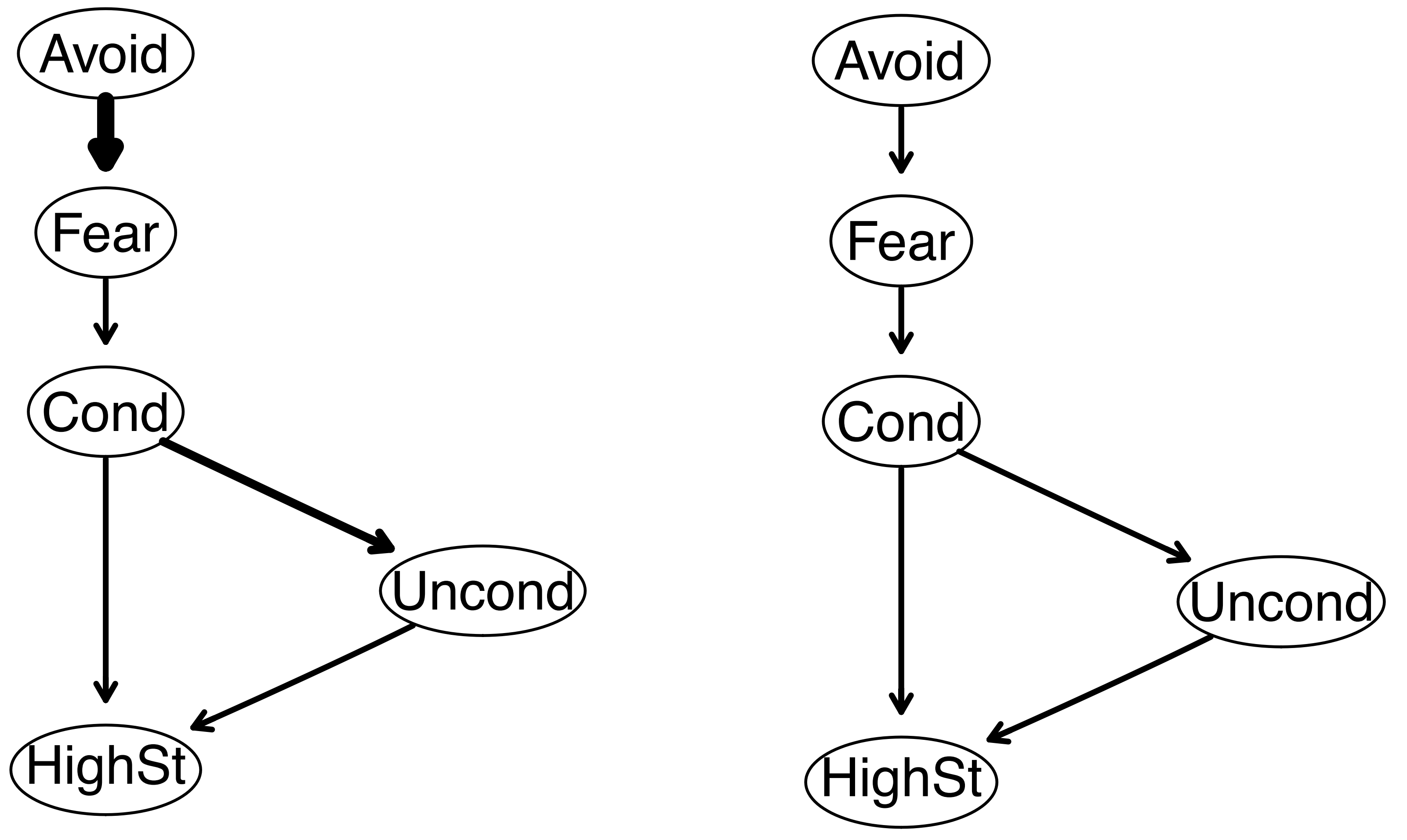